\title{
Control of Minimally Persistent Leader-Remote- Follower and Coleader Formations in the Plane
}

\author{
Tyler H. Summers, Member, IEEE, Changbin Yu, Senior Member, IEEE, Soura Dasgupta, Fellow, IEEE, and \\ Brian D. O. Anderson, Life Fellow, IEEE
}

\begin{abstract}
This paper solves an $n$-agent formation shape control problem in the plane. The objective is to design decentralized control laws so that the agents cooperatively restore a prescribed formation shape in the presence of small perturbations from the prescribed shape. We consider two classes of directed, cyclic information architectures associated with so-called minimally persistent formations: leader-remote-follower and coleader. In our framework the formation shape is maintained by controlling certain interagent distances. Only one agent is responsible for maintaining each distance. We propose a decentralized control law where each agent executes its control using only the relative position measurements of agents to which it must maintain its distance. The resulting nonlinear closed-loop system has a manifold of equilibria, which implies that the linearized system is nonhyperbolic. We apply center manifold theory to show local exponential stability of the desired formation shape. The result circumvents the non-compactness of the equilibrium manifold. Choosing stabilizing gains is possible if a certain submatrix of the rigidity matrix has all leading principal minors nonzero, and we show that this condition holds for all minimally persistent leader-remote-follower and coleader formations with generic agent positions. Simulations are provided.
\end{abstract}

Index Terms-Center manifold theory, formation shape control, graph persistence, graph rigidity.

\section{INTRODUCTION}

$\mathbf{T}$ HERE has been increased interest recently in cooperative control of autonomous vehicle formations and mobile sensor networks. Ongoing rapid advancements in computation, communication, sensing, and control technologies have made it possible to imagine systems in which multiagent cooperation allows unprecedented new sensing capabilities when

Manuscript received August 05, 2009; revised May 19, 2010, May 20, 2010, February 15, 2011; accepted February 18, 2011. Date of publication April 25, 2011; date of current version December 07, 2011. Thus work was supported by the Australian-American Fulbright Commission, by the Australian Research Council through an Australian Postdoctoral Fellowship a Discovery Project DP-0877562, by DP-0877562 and National ICT Australia-NICTA (the NICTA is funded by the Australian Government as represented by the Department of Broadband, Communications and the Digital Economy and the Australian Research Council through the ICT Centre of Excellence program), and by National Science Foundation (NSF) grants ECS-0622017, CCF-072902, and CCF-0830747. Recommended by Associate Editor M. Egerstedt.

T. H. Summers was with the Australian National University, Canberra, ACT 0200, Australia. He is now with the Automatic Control Laboratory, ETH Zürich, 8092 Zürich, Switzerland (e-mail: tylerhsummers@gmail.com).

C. Yu and B. D. O. Anderson are with the Research School of Information Sciences and Engineering, Australian National University, Canberra, ACT 0200, Australia (e-mail: brad.yu@ anu.edu.au).

S. Dasgupta is with the Department of Electrical and Computer Engineering, University of Iowa, Iowa City, IA 52242 USA.

Color versions of one or more of the figures in this paper are available online at http://ieeexplore.ieee.org.

Digital Object Identifier 10.1109/TAC.2011.2146890 compared to what can be achieved with a single agent. The motivation for studying such systems comes from both the ubiquitous potential for applications and the interesting technical challenges that such systems present. Applications include teams of unmanned aircraft for military reconnaissance missions, satellite formations for deep space imaging, and submarine swarms for oceanic exploration. For large formations, an overarching requirement and key technical challenge is that the formation operates in a decentralized fashion, where each agent operates using only local information.

A fundamental task for autonomous vehicle formations is formation shape control. Precisely controlled formations can maintain mobile sensing agents in optimal sensing configurations. In this paper, we study an $n$-agent formation shape control problem. The objective is to design decentralized control laws for each agent to restore a desired formation shape in the presence of small perturbations from the desired shape.

A critical feature of the formation shape control problem is the information architecture, i.e., precisely what variables are sensed and what are controlled. We use certain relative position measurements to achieve shape maintenance. A key distinction in the literature involves whether the controlled variables are a set of relative positions or a set of interagent distances (if $p_{i} \in \Re^{2}$ and $p_{j} \in \Re^{2}$ are the positions of agents $i$ and $j$, respectively, then the relative position of agent $j$ with respect to agent $i$ is $p_{j}-p_{i}$ whereas the interagent distance between agents $i$ and $j$ is $\left.\left\|p_{j}-p_{i}\right\|\right)$. When agents actively control relative positions, the formation shape maintenance problem can be cast as a consensus problem [1]-[4]. A consensus problem is to design decentralized control laws to drive the agents toward agreement on certain quantities of interest, e.g., relative position. In consensus problems, a crucial role is played by the graph Laplacian. On the other hand, when agents actively control interagent distances, then the graph rigidity [5] of the information architecture becomes the crucial concept for the formation shape maintenance problem, with the rigidity matrix playing a crucial role in the stability analysis [6]-[12]. An advantage of controlling distances is that the relative position measurements for each agent can be taken in an arbitrary coordinate basis, whereas controlling relative positions and using a consensus algorithm requires relative position measurements in a global coordinate basis.

A further delineation in the literature for rigidity-based formation control involves whether the information architecture is undirected or directed. In an undirected formation, the task of controlling a particular interagent distance is shared by both of the involved agents. Undirected formations with gradient-based control laws are studied in [12]. In a directed formation, the task of controlling a particular interagent distance is given to only 
one of the involved agents. This has the advantage of halving the number of links in the information architecture. Directed formations are studied in [8]-[11], [13] and are the focus of this paper. In [8], global stability analysis for a cyclic three-agent formation is provided; the control law is generalized in [9]. Formations of nonholonomic robots are considered in [13].

The concept of rigidity for directed graphs is not a simple transposition of rigidity for undirected graphs; therefore, the term persistence is used for directed graphs to distinguish from the undirected notion [14]. Persistence includes rigidity, but also requires a further condition called constraint consistence that precludes certain directed information flow patterns [15]. In directed formations, it becomes possible to have cycles in the information architecture. Acyclic formations engender a triangular decomposition that makes stability much easier to deal with [10]. Cyclic formations present possibilities of instability and the challenge of controlling it. This paper considers minimally persistent formations (persistent formations with the minimum number of links in the information architecture), which fall into three categories: Leader-First-Follower (LFF), LeaderRemote-Follower (LRF), and Coleader. In [11], Yu et al. consider minimally persistent LFF formations in the plane with $c y$ cles. We emphasize that LFF does not imply acyclic; acyclic LFF formations are considered in [10]. Yu et al. present decentralized nonlinear control laws to restore formation shape in the presence of small distortions from the desired shape. They show that choosing stabilizing control gains is possible if a certain submatrix of the rigidity matrix has all leading principal minors nonzero and further prove that all minimally persistent LFF formations generically obey this principal minor condition.

The main contribution of this paper is to minimally persistent $L R F$ and coleader formations. This is nontrivial because the nonlinear closed-loop system has a manifold of equilibria, which implies that the linearized system is nonhyperbolic. Center manifold theory is thus required in the stability analysis. For LFF formations, Yu et al. obtain a hyperbolic system via the choice of a particular global coordinate system and prove local exponential stability of the formation shape through eigenvalue analysis. For LRF and coleader formations, such a choice of global coordinate system is not possible. Accordingly, we apply a new result based on center manifold theory to show local exponential stability of the desired formation shape. In [12], Krick et al. also use center manifold theory, but their analysis is restricted to undirected formations. A further key challenge of the argument, in contrast to that of [12], is to circumvent the non-compactness of the equilibrium manifold. We show that it is again possible to choose stabilizing control gains whenever a certain submatrix of the rigidity matrix has all leading principal minors nonzero and show that this condition holds for all LRF and coleader formations. This paper and [11] together constitute a solution to the formation shape maintenance problem for all minimally persistent formations. The practical utility of LRF and coleader formations remains, however, a question for future research.

The paper is organized as follows. Section II presents background on the structure of minimally persistent formations and center manifold theory. Section III presents a new center manifold theory result for systems with an equilibrium manifold. In Section IV, we describe the nonlinear equations of motion and show how center manifold theory can be applied to prove that the desired formation shape is locally exponentially stable. In Section V, we show that the principal minor condition holds for all LRF and coleader formations. In Section VI, we illustrate the results with numerical simulations. Section VII gives concluding remarks and future research directions.

\section{BACKGROUND}

In this section, we review the structure of information architectures for minimally persistent formations, and center manifold theory, which offers tools for analyzing stability of dynamical systems near nonhyperbolic equilibrium points. Drawing from [14], we briefly review the concepts of rigidity and persistence in the plane; for a complete treatment in the plane see [14] and for extensions to higher dimensions see [15].

\section{A. Rigid and Persistent Formations}

1) Rigidity: The information architecture of a formation is represented by a graph $G(V, E)$ where the vertex set $V$ represents the agents and the edge set $E$ represents the set of interagent distances to be controlled to maintain formation shape. A representation $p: V \rightarrow \Re^{2 n}$ is a function which assigns to each vertex a position in the plane, and we call $p_{i} \in \Re^{2}$ the position of vertex $i$. Consider all continuous motions such that the distances between any two vertices connected by an edge remains constant. The graph is called rigid if for almost all ${ }^{1}$ representations, every such motion preserves the distance between every vertex pair. A formation is an information architecture together with a representation $F=(G, p)$ and is called rigid if $G$ is a rigid graph.

Rigidity is a generic property; that is, for a formation with generic agent positions, it is not the particular agent positions that matter in determining rigidity, but rather the distribution of the edges among the graph vertices. This leads us to the following paraphrase of Laman's well-known combinatorial characterization of rigid graphs in the plane [18].

Theorem 1 ([18]): A graph $G(V, E)$ is rigid iff there is a subgraph $G^{\prime}\left(V, E^{\prime}\right), E^{\prime} \subset E$ that satisfies the following:

- $\left|E^{\prime}\right|=2|V|-3$.

- For any $V^{\prime} \subset V$ and the associated induced subgraph $G^{\prime \prime}\left(V^{\prime}, E^{\prime \prime}\right)$ of $G^{\prime}$ with $E^{\prime \prime} \subset E^{\prime}$, there holds $\left|E^{\prime \prime}\right| \leq$ $2\left|V^{\prime}\right|-3$.

The first condition gives the minimum number of edges required for a rigid graph: given $|V|$ vertices, one must have at least $2|V|-3$ edges. The second condition gives the manner in which a minimum set of edges must be distributed amongst the vertices to ensure rigidity. A graph is called minimally rigid if it is rigid and has exactly $2|V|-3$ edges.

There is a separate linear algebraic way to characterize rigidity involving the rigidity matrix. Let $F(G, p)$ be a formation in the plane and $p_{i} \in \Re^{2}$ be the position of agent $i$. Let $r: \Re^{2|V|} \rightarrow \Re^{|E|}$ be the rigidity function defined by

\footnotetext{
${ }^{1}$ The term almost all is referring here to generic representations. A representation is called generic if the set of coordinates of agent positions is algebraically independent over the rationals. Non-generic representations correspond to special agent configurations, e.g., all agents are collinear. The fact that rigidity is a generic property is nontrivial; for discussion on use of the terms "generic" and "almost all" in this context, see [5], [16], [17]
} 


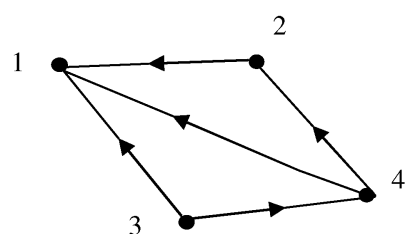

(a)

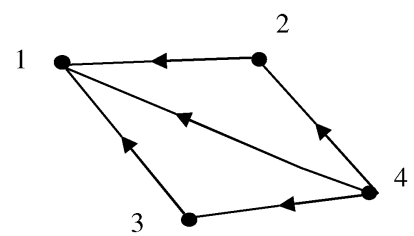

(b)
Fig. 1. Constraint consistent and non-constraint consistent graphs with the same underlying undirected graph.

$$
r(p)=\frac{1}{2}\left[\ldots,\left\|p_{j}-p_{k}\right\|^{2}, \ldots\right]^{T}
$$

where the $i$ th entry of $r, v i z .\left\|p_{j}-p_{k}\right\|^{2}$, corresponds to the edge $e_{i} \in E$ connecting vertex $j$ to vertex $k$ in $G$. The rigidity matrix $R(p) \in \Re^{|E| \times 2|V|}$ is given by the Jacobian of $r$ and has the following structure. The columns of $R$ are regarded as $|V|$ sets of two columns, with each set of two columns corresponding to a vertex of $G$, i.e., columns $2 i-1$ and $2 i$ correspond to vertex $i$. Each row of $R$ corresponds to an edge of $G$. In the $i$ th row corresponding to the edge connecting vertices $j$ and $k$, all entries zero except in columns $2 j-1,2 j, 2 k-1,2 k$, which are $x_{j}-x_{k}$, $y_{j}-y_{k}, x_{k}-x_{j}$, and $y_{k}-y_{j}$, respectively, with $\left(x_{j}, y_{j}\right)$ denoting the coordinates of agent $j$. We have the following result from [5].

Theorem 2 ([5]): Let $F(G, p)$ be a formation in the plane with generic representation $p$ and let $R(p)$ be the associated rigidity matrix. Then $F$ is rigid iff the rank of $R(p)$ is $2|V|-3$.

Thus, a rigid formation has at least $2|V|-3$ well-distributed edges and has a rigidity matrix with full row rank. Further, the dimension of the null space is exactly three. The vectors in the null space correspond to a set of infinitesimal displacements of the agents that preserve the formation shape, with the three independent displacements spanning the null space corresponding to translation in two directions and rotation of the entire formation.

2) Persistence: There are two possible ways that a particular interagent distance can be controlled. Either the two involved agents share the responsibility, resulting in an undirected formation, or only one of the involved agents is given the responsibility, resulting in a directed formation. Directed formations have the advantage of reducing the sensing/communication requirements by half, and naturally lend themselves to leader-follower formations that are prevalent in the literature. In a directed formation, a direction is assigned to every edge in $E$ with an outward arrow from the agent responsible for controlling the interagent distance, so $G$ becomes a directed graph.

The concept of rigidity for directed graphs is not a simple transposition of rigidity for directed graphs; therefore, the term persistence is used for directed graphs to distinguish from the undirected notion. In order to preclude situations where the formation shape maintenance task becomes impossible for directed information flows, a further condition called constraint consistence is required in addition to rigidity. Examples of a constraint consistent and non-constraint consistent graph are shown in Fig. 1, taken from [11]. In the right-hand graph, agent 1 is free to move in the plane while agents 2 and 3 can move on circles around agent 1; thus, it may be impossible for agent 4 to maintain all of its distance constraints simultaneously. For further discussion and rigorous definitions, see [11], [14], and [15].

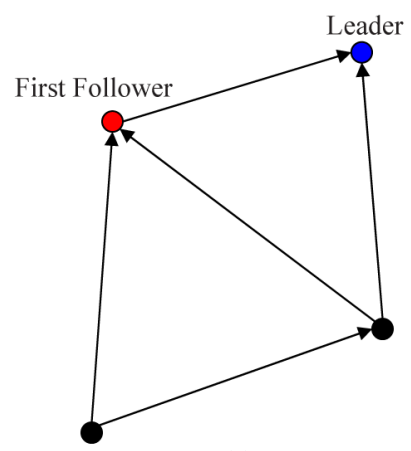

(a)

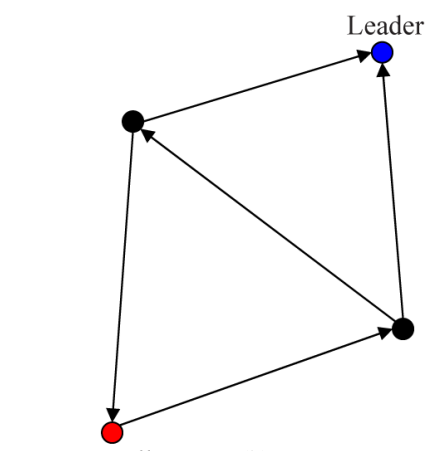

Remote Follower (b)
Fig. 2. Examples of LFF and LRF formations with four agents. (a) In LFF formations, the one-DOF agent is connected to the leader, and (b) in LRF formations the one-DOF agent is not connected to the leader.

3) Minimally Persistent Formations: Our exclusive focus in this paper will be on minimally persistent formations. We have the following basic result from [14].

Theorem 3 ([14]): Let $G(V, E)$ be a directed graph in the plane with at least two vertices. Then $G$ is minimally persistent iff the underlying undirected graph is minimally rigid, which has precisely $2|V|-3$ edges, and no vertex has more than two outgoing edges.

This means in particular that one of the following happens for minimally persistent formations.

- Type (A) [Leader-First-Follower (LFF)]: One agent known as the leader has no outgoing edge, i.e., zero distances to maintain and thus two degrees of freedom (DOF). Another known as the first follower has one out going edge to the leader, i.e., one distance to maintain and thus one DOF. The remaining, ordinary followers have two outgoing edges each, i.e., two distances to maintain and thus zero DOF.

- Type (B) [Leader-Remote-Follower (LRF)]: One agent known as the leader has no outgoing edge (two DOF), another known as the remote follower has one outgoing edge to an agent other than the leader (one DOF), and the remaining ordinary followers have two outgoing edges each (zero DOF).

- Type (C) [Coleader]: three agents (known as coleaders), have one outgoing edge each (one DOF each) and all others (known as ordinary followers), have exactly two such edges each (zero DOF). Fig. 2 illustrates examples LFF and LRF formations. Fig. 3 illustrates examples of coleader formations with differing information flow patterns and coleader connectivity.

In directed formations, it becomes possible to have cycles in the information architectures. LFF formations can be either cyclic or acyclic, and LRF and coleader formations are inherently cyclic (see Theorem 5 of [14]). A cyclic information architecture presents a possibility of instability and the challenge of controlling it.

When might the last two structures be used? There is currently no straightforward measure like a controllability gramian that might give guidance as to preferred information structures, and such a measure might well give guidance. Apart from that, one can readily contemplate intuitively the potential attractiveness of a cyclic coleader pattern in the case of a formation three of whose agents define a triangle, with all other agents in the interior of the triangle. The three agents defining the triangle 


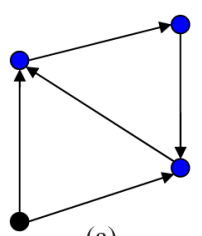

(a)

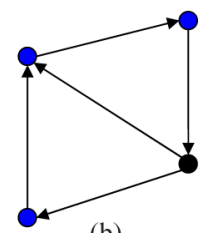

(b)

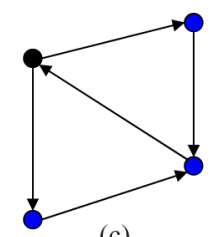

(c)

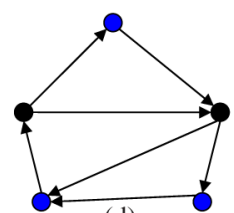

(d)

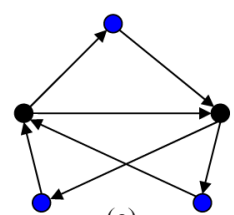

(e) indicates coleader

Fig. 3. Examples of coleader formations with connected coleaders. (a) Cyclic coleaders. (b) Inline coleaders. (c) v-coleaders. Examples of coleader formations with non-connected coleaders. (d) One-two coleaders. (e) Distributed coleaders. Each coleader has only one interagent distance to maintain and so has only one outgoing arrow.

would in some sense confine and lead the rest of the formation. The motion of the three agents in this case can be analyzed as in [8], and would be independent of the motion of other agents, though not conversely. In relation to a leader-remote-follower structure, one could conjecture its relevance for control of a formation whose shape was long and thin. At one end of the formation, the leader would move in the direction of a target. At the other end of the formation, the freedom of the remote follower would be used to rotate the formation, thereby aligning its longer axis with the direction of target motion.

The distinction between LFF, LRF, and coleader formations is important in the stability analysis for the formation shape maintenance control laws. In particular, for LFF formations it is possible to define a global coordinate basis to obtain a hyperbolic reduced-order system in which local stability can be ascertained via eigenvalue analysis of the linearized system [11]. This is so because in the framework of both [11] and this paper, after its "small" initial move, the leader stops moving. Thereafter, the algorithm of [11] forces the first follower to move in the direction of the leader. Thus, the direction of movement of the first follower in the LFF framework is fixed. This direction critically defines the stated coordinate basis in [11]. In contrast, for LRF formations the direction associated with the remote follower's DOF is not fixed in space since it is following an agent other than the leader to satisfy its distance constraint. Similarly, for coleader formations the directions associated with the coleader DOFs are not fixed in space. Thus, the device used in [11] to obtain a global coordinate system that provides a hyperbolic reduced-order system no longer applies. Consequently, one cannot draw conclusions about the local stability of the nonlinear system near the desired formation shape by analyzing the linearized system alone; more sophisticated techniques are needed. Center manifold theory provides tools for determining stability near nonhyperbolic equilibrium points.

In contrast to [11], [12] does use center manifold theory to establish stability of the closed-loop system, but their analysis is restricted to undirected graphs. It is possible to work with a reduced-order system, using the fact that for the laws in question, the centroid of the formation is stationary. This allows the full-order system to be reduced by 2 in dimension, and leaves one zero eigenvalue for the linearized system. A critical feature of the center manifold analysis of [12] is the observation that the set of equilibria of this reduced-order system is then compact. For LRF formations, one can fix the position of the leader to obtain a compact set of equilibria for a reduced-order system. However, this is not possible in the coleader setting. In either case, separate aspects of center manifold theory have to be used to establish a related de facto compactness condition.

\section{B. Center Manifold Theory}

Center manifold theory deals with stability of nonhyperbolic equilibrium points of a nonlinear system (that is, equilibrium points about which the linearization has one or more eigenvalues with zero real part). Effectively, the theory allows one to reduced the dimension of the nonlinear system. The motions tend asymptotically toward trajectories on the center manifold rather than to a point. Standard treatments of center manifold theory can be found in e.g., [19]-[21]. These concentrate on isolated equilibria. In the formation shape maintenance problem, the dynamic system has a manifold of non-isolated equilibrium points corresponding to the desired formation shape that for the coleader case is not even compact. In [22], Malkin proves a local stability result where trajectories converge to a point on an equilibrium manifold. More general results for equilibrium manifolds are presented by Aulbach in [23]. In [12], Krick et al. emphasize the importance of compactness for proving stability of the entire equilibrium manifold. Here, we state a new result for stability of equilibrium manifolds and offer a concise proof using center manifold theory.

Consider the nonlinear autonomous dynamic system

$$
\dot{x}=f(x), \quad x \in \Re^{n}
$$

where the function $f$ is $\mathbf{C}^{r}, r \geq 2$ almost everywhere including a neighborhood of the origin. Suppose the origin is an equilibrium point and that the Jacobian of $f$ (we will use the notation $J_{f}(x)$ ) at the origin has $m$ eigenvalues with zero real part and $n-m$ eigenvalues with negative real part. Then (2) can be locally transformed into the following form:

$$
\begin{aligned}
& \dot{\theta}=A_{c} \theta+g_{1}(\theta, \rho) \\
& \dot{\rho}=A_{s} \rho+g_{2}(\theta, \rho), \quad(\theta, \rho) \in \Re^{m} \times \Re^{n-m}
\end{aligned}
$$

where $A_{c}$ is a matrix having eigenvalues with zero real parts, $A_{s}$ is a matrix having eigenvalues with negative real parts, and the functions $g_{1}$ and $g_{2}$ satisfy

$$
\begin{array}{ll}
g_{1}(0,0)=0, & J_{g_{1}}(0,0)=0 \\
g_{2}(0,0)=0, & J_{g_{2}}(0,0)=0 .
\end{array}
$$

Definition 1: An invariant manifold is called a center manifold for (3) if it can be locally represented as follows;

$$
W^{c}(0)=\left\{(\theta, \rho) \in U \subset \Re^{m} \times \Re^{n-m} \mid \rho=h(\theta)\right\}
$$

for some sufficiently small neighborhood of the origin $U$ where the function $h$ satisfies $h(0)=0$ and $J_{h}(0)=0$.

We have the following standard result. 
Theorem 4 ([21]): Consider (3) where $A_{c}$ has eigenvalues with zero real part, $A_{s}$ has eigenvalues with negative real part, and $g_{1}$ and $g_{2}$ satisfy (4). There exists a $\mathbf{C}^{r}$ center manifold for (3) with local representation function $h: \Re^{m} \rightarrow \Re^{n-m}$. The dynamics of (3) restricted to any such center manifold is given by the following $m$-dimensional nonlinear system for $\xi$ sufficiently small

$$
\dot{\xi}=A_{c} \xi+g_{1}(\xi, h(\xi)) \quad \xi \in \Re^{m} .
$$

If the origin of (6) is stable (asymptotically stable) (unstable), then the origin of (3) is stable (asymptotically stable) (unstable). Suppose the origin of (6) is stable. Then if $(\theta(t), \rho(t))$ is a solution of (3) for sufficiently small $(\theta(0), \rho(0))$, there is a solution $\xi(t)$ of (6) such that as $t \rightarrow \infty$

$$
\begin{aligned}
& \theta(t)=\xi(t)+O\left(e^{-\gamma t}\right) \\
& \rho(t)=h(\xi(t))+O\left(e^{-\gamma t}\right)
\end{aligned}
$$

where $\gamma$ is a positive constant.

This result shows that in order to determine stability near the nonhyperbolic equilibrium point of (2), one can analyze a reduced-order system, viz. (6). If the origin of the reducedorder system (6) is stable, then the solutions of the original system converge exponentially to a trajectory on the center manifold. This result however only applies when the nonhyperbolic equilibrium point is isolated. In our formation shape control problem, we will see that there is a smooth manifold of equilibrium points. This requires a new center manifold result, which we provide in the following section.

\section{Center Manifold Result for a System WITH AN EQUILIBRIUM MANIFOLD}

We have the following result when there is a manifold of equilibria, and we will contrast it with the result of [12]. There are two essential differences with the result of [12], both related to the fact that [12] deals with undirected formations and the simplifications they engender.

First the undirected nature of the formations renders the center manifold underlying [12] compact. This is in fact quite crucial to the argument in [12]. Ultimately the goal of [12] and indeed our goal is to demonstrate exponential convergence to the center manifold. The lack of compactness of this manifold, that unlike the setting of [12] is the case here, causes difficulties. $^{2}$

The second difference stems from the fact that the undirected nature of the formation considered in [12] makes the entire center manifold, under the control law of [12], exponentially stable. In the directed formation case considered here that does not necessarily happen. This requires a fundamentally new result provided in Theorem 5 below. Observe that although the theorem postulates and proves the existence of a center manifold, it makes no explicit compactness assumptions, in contrast to [12].

Theorem 5: Consider (2) with $f \in \mathbf{C}^{r}, r \geq 2$ almost everywhere including a neighborhood of the origin. Suppose there is a

\footnotetext{
${ }^{2}$ Saying that a manifold is locally exponentially stable means that there is a single exponent $\gamma$ such that all trajectories converge to the manifold from a sufficiently small neighborhood at least as fast as $e^{-\gamma t}$. One could envisage a non-compact manifold where any single trajectory approaches the manifold exponentially fast but no single $\gamma$ could be found applicable to all trajectories.
}

smooth $m$-dimensional $(m>0)$ manifold of equilibrium points $M_{1}=\left\{x \in \Re^{n} \mid f(x)=0\right\}$ for (2) that contains the origin. Suppose at the origin the Jacobian of $f$ has $m$ eigenvalues with zero real part and $n-m$ eigenvalues with negative real part. Then we have the following:

- $M_{1}$ is a center manifold for (2);

- there are compact neighborhoods $\Omega_{1}$ and $\Omega_{2}$ of the origin such that $M_{2}=\Omega_{2} \cap M_{1}$ is locally exponentially stable and for each $x(0) \in \Omega_{1}$ there is a point $q \in M_{2}$ such that $\lim _{t \rightarrow \infty} x(t)=q$.

We shall prove Theorem 5 with the aid of the following lemma.

Lemma 1: Consider (3) where $A_{c}$ has eigenvalues with zero real part, $A_{s}$ has eigenvalues with negative real part, and $g_{1}$ and $g_{2}$ satisfy (4). Suppose that the equilibrium set is a smooth $m$-dimensional manifold $M_{1}$. Then there exists a smooth function $h_{1}: \Re^{m} \rightarrow \Re^{n-m}$ such that $h_{1}(0)=0, J_{h_{1}}(0)=0$ and in a suitably small neighborhood $U$ of the origin, the equilibrium set can be represented as $\rho=h_{1}(\theta)$.

Proof: Consider the equation

$$
\tilde{g}_{2}(\theta, \rho)=A_{s} \rho+g_{2}(\theta, \rho)=0
$$

As $A_{s}$ is nonsingular and (4) holds, then by the implicit function theorem, for any suitably small $\theta$, there exists $\rho$, expressible as $\rho=h_{1}(\theta)$, for a smooth $h_{1}$ satisfying $h_{1}(0)=0, J_{h_{1}}(0)=0$, and such that (8) holds.

Note that to prove $\left(\theta, \rho=h_{1}(\theta)\right)$ lies on the equilibrium manifold, we must show in addition to (7) that the following equation holds:

$$
A_{c} \theta+g_{1}\left(\theta, h_{1}(\theta)\right)=0 \text {. }
$$

To do this, we now appeal to the fact that $M_{1}$ is an $m$-dimensional manifold. As a result, there exists a neighborhood $W$ of the origin in $R^{m}$ and a diffeomorphism $\phi=\left(\phi_{1}, \phi_{2}\right)$ such that for $w \in W$

$$
\left[\begin{array}{l}
\phi_{1} \\
\phi_{2}
\end{array}\right]: w \rightarrow\left[\begin{array}{l}
\theta \\
\rho
\end{array}\right] \in M_{1} \cap U .
$$

Then the set of equilibria include $\theta=\phi_{1}(w), \rho=\phi_{2}(w)$ for all $w \in W$. Because an equilibrium point necessarily satisfies (8), it is necessary that the function $h_{1}(\cdot)$ identified above is such that $\phi_{2}(w)=h_{1}\left(\phi_{1}(w)\right) \forall w \in W$. The mapping from $w$ to the set of equilibria is then $\left(\phi_{1}(w), h_{1}\left(\phi_{1}(w)\right)\right)$, and it is invertible, being a diffeomorphism. Therefore, $\phi_{1}: w \rightarrow \theta$ itself must be invertible. Let $w=\psi(\theta)$. Then the equilibria are $\left(\phi_{1}\left(\psi(\theta), h_{1}\left(\phi_{1}(\psi(\theta))\right)=\left(\theta, h_{1}(\theta)\right)\right.\right.$. This establishes the claim of the lemma.

Proof of Theorem 5: By assumption, there exists a similarity transformation $Q$ and a neighborhood $U$ of the origin, such that for each $\bar{x} \in U \cap M_{1}$, we can write

$$
Q J_{f}(\bar{x}) Q^{-1}=\left[\begin{array}{cc}
A_{c} & 0 \\
0 & A_{s}
\end{array}\right] .
$$

Therefore, for any equilibrium point $\bar{x} \in U \cap M_{1}$, we can write (2) in the form given by (3). The first part of the theorem is then immediate, for $M_{1}$, being an equilibrium manifold, is invariant, and Lemma 1 demonstrates the local representation condition required for $M_{1}$ to be a center manifold. 
Now, we turn to the second part of the theorem. Since $M_{1}$ is a center manifold, the dynamics restricted to $M_{1}$ are stationary. Therefore, from (6) we have $\dot{\xi}=0$, which implies $\xi(t)=\xi(0)$ $\forall t$. Thus, the reduced-order system is stable. Theorem 4 then mandates that the origin is locally stable. This together with the stated conditions of the Jacobian, ensures that there exist compact neighborhoods $\Omega_{1}$ and $\Omega_{2}$ of the origin such that a) all trajectories starting in $\Omega_{1}$ remain in $\Omega_{2}$,b) with $M_{2}$ defined in the theorem statement, the Jacobian of $f$ has $m$ eigenvalues with zero real part and $n-m$ eigenvalues with negative real part everywhere in $M_{2}$, and c) there is a $\gamma>0$ such that for each $\bar{x} \in M_{2}, \operatorname{Re}\left[e i g\left(A_{s}(\bar{x})\right)\right]<-\gamma$. (The compactness of $M_{2}$ is crucial.) The exponential convergence mandated by Theorem 4 implies that $\Omega_{1}$ and $\Omega_{2}$ can be chosen to have the additional property that trajectories starting in $\Omega_{1}$ converge exponentially to a point on the center manifold and thus to a point in $M_{2}$ (though not necessarily to the origin).

Finally, this argument is valid for any point in $M_{2}$. For each point in $M_{2}$, there is an open neighborhood of attraction $U_{i}$. Further, $M_{2}$ is a compact subset of $M_{1}$. By the Heine-Borel Theorem, there is a finite subcover $\Omega_{3}$ of $M_{2}$ constructible from a finite union of these neighborhoods. The smallest exponential convergence rate associated with the neighborhoods of the finite subcover is valid to bound the speed of convergence for all trajectories starting in $\Omega_{3}$. The final part immediately follows. $\square$

In the formation shape maintenance problem, the manifold of equilibria will correspond to formation positions with the desired shape. In the plane, the manifold is three-dimensional due to the three possible Euclidean motions of the formation in the plane (two translational and one rotational). In the following section, we develop equations of motion and apply Theorem 5 to show local exponential stability of the desired shape.

\section{EQuATIONS OF Motion}

In this section, we present equations of motion for the formation shape maintenance problem and study the local stability properties of the desired formation shape. Suppose the formation is initially in the desired shape. Then the position of each agent is perturbed by a small amount and all agents (except for the leader in a LRF formation) move under distance control laws to meet their distance specifications in order to restore the desired formation shape. This shape is realized by every point on a smooth three-dimensional equilibrium manifold. For LRF and coleader formations, we show that a direct application of Theorem 5 proves local exponential convergence to the invariant manifold, whether or not the equilibrium manifold is compact.

\section{A. Nonlinear Equations of Motion}

Consider a minimally persistent formation $F(G, p)$ of $n$ agents in the plane. For LRF formations the leader and remote follower are agents $n$ and $n-1$, respectively. For coleader formations the coleaders are agents $n-2, n-1$, and $n$. Recall the rigidity function $r$ from (1) and let $d=\left[\ldots, d_{j k}^{* 2}, \ldots\right]$ represent a vector of the squares of the desired distances corresponding to each edge of $G$. We assume that there exist agent positions $p$ such that $p=r^{-1}(d)$, i.e., the set of desired interagent distances is realizable. Formation shape is controlled by controlling the interagent distance corresponding to each edge.
Following [11] and [12], we adopt a single integrator model for each agent:

$$
\dot{p}_{i}=u_{i}
$$

Consider an ordinary follower agent denoted by $i$ that is required to maintain constant distances $d_{i j}^{*}$ and $d_{i k}^{*}$ from agents $j$ and $k$, respectively, and can measure the instantaneous relative positions of these agents. We use the same law as in [11] for ordinary followers:

$$
u_{i}=K_{i}\left(p_{i}^{*}-p_{i}\right)=K_{i} f_{i}\left(p_{j}-p_{i}, p_{k}-p_{i}, d_{i j}^{*}, d_{i k}^{*}\right)
$$

where $K_{i}$ is a gain matrix and $p_{i}^{*}$ is the closest instantaneous target position for agent $i$ in which its distances from agents $j$ and $k$ are correct. Since the perturbations from the desired shape are small, these instantaneous target positions are well-defined and unique. For the remote follower $(i=n-1)$ or the coleaders $(i=n-2, n-1, n)$, we have

$$
\begin{aligned}
u_{i} & =K_{i}\left(p_{i}^{*}-p_{i}\right) \\
& =K_{i} \frac{\left\|p_{j(i)}-p_{i}\right\|-d_{i j(i)}^{*}}{\left\|p_{j(i)}-p_{i}\right\|}\left(p_{j(i)}-p_{i}\right)
\end{aligned}
$$

where $K_{i}$ is a gain matrix and agent $j(i)$ is the agent from which the remote follower or coleader $i$ is maintaining the constant distance $d_{i j(i)}^{*}$ (note that each coleader may follow a different agent). For the leader, we have

$$
\dot{p}_{n}=0 \text {. }
$$

For a LRF formation, (13), (14), and (15) represent the dynamics of the autonomous closed-loop system, which may be written in the form

$$
\dot{p}=\left[\begin{array}{c}
f_{L R F}(p) \\
0
\end{array}\right]
$$

where $f_{L R F}: \Re^{2 n} \rightarrow \Re^{2 n-2}$ is smooth almost everywhere including a neighborhood of the desired formation. For a coleader formation, (13) and (14) represent the dynamics of the autonomous closed-loop system, which may be written in the form

$$
\dot{p}=f_{C}(p)
$$

where $f_{C}: \Re^{2 n} \rightarrow \Re^{2 n}$ is smooth almost everywhere including a neighborhood of the desired formation.

There is a smooth manifold of equilibria for (16) and (17) given by

$$
\Psi=\left\{p \in \Re^{2 n} \mid p=r^{-1}(d)\right\}
$$

corresponding to formations where all distance constraints are satisfied. The manifold $\Psi$ is a three-dimensional manifold because a formation with correct distances has three degrees of freedom associated with the planar Euclidean motions (two for translation and one for rotation). Given these degrees of freedom, it is evident that $\Psi$ is not compact. For LRF formations, we can define a reduced-order system by fixing the position of the leader and obtain a compact equilibrium manifold. 


\section{B. Linearized Equations}

We represent the position of the formation as $p(t)=\delta p(t)+$ $\bar{p}$, where $\bar{p}$ is any equilibrium position with desired shape close to the perturbed formation, and the displacements $\delta p(t)$ are assumed to be small. In particular, for agent $i$ we have $p_{i}(t)=$ $\delta p_{i}(t)+\bar{p}_{i}$. Let $p_{i}(t)=\left[x_{i}(t), y_{i}(t)\right]^{T}, \bar{p}_{i}=\left[\bar{x}_{i}, \bar{y}_{i}\right]^{T}$, and $\delta p_{i}(t)=\left[\delta x_{i}(t), \delta y_{i}(t)\right]^{T}$ in a global coordinate system to be defined later.

From [11], the linear part for the ordinary followers is given by

$$
\left[\begin{array}{l}
\dot{\delta} x_{i} \\
\dot{\delta} y_{i}
\end{array}\right]=K_{i} R_{e i}^{-1} R_{i j, i k}\left[\begin{array}{c}
\delta x_{i} \\
\delta y_{i} \\
\delta x_{j} \\
\delta y_{j} \\
\delta x_{k} \\
\delta y_{k}
\end{array}\right]
$$

where

$$
R_{e i}=\left[\begin{array}{l}
\left(\bar{p}_{j}-\bar{p}_{i}\right)^{T} \\
\left(\bar{p}_{k}-\bar{p}_{i}\right)^{T}
\end{array}\right]
$$

and

$$
R_{i j, i k}=\left[\begin{array}{ccc}
\left(\bar{p}_{i}-\bar{p}_{j}\right)^{T} & \left(\bar{p}_{j}-\bar{p}_{i}\right)^{T} & 0 \\
\left(\bar{p}_{i}-\bar{p}_{k}\right)^{T} & 0 & \left(\bar{p}_{k}-\bar{p}_{i}\right)^{T}
\end{array}\right]
$$

The matrix $R_{e i}$ is nonsingular because the equilibrium positions of $i, j, k$ are not collinear (collinearity would violate minimal rigidity). Similarly, the linearized equation for the remote follower $(i=n-1)$ or the coleaders $(i=n-2, n-1, n)$ is given by

$$
\left[\begin{array}{c}
\dot{\delta} x_{i} \\
\dot{\delta} y_{i}
\end{array}\right]=K_{i} R_{e i}^{-1} R_{i j(i), 00}\left[\begin{array}{c}
\delta x_{i} \\
\delta y_{i} \\
\delta x_{j(i)} \\
\delta y_{j(i)}
\end{array}\right]
$$

where $K_{i}$ is a $2 \times 2$ gain matrix,

$$
R_{e i}=\left[\begin{array}{cc}
\bar{x}_{j(i)}-\bar{x}_{i} & \bar{y}_{j(i)}-\bar{y}_{i} \\
\bar{y}_{i}-\bar{y}_{j(i)} & \bar{x}_{j(i)}-\bar{x}_{i}
\end{array}\right]
$$

and

$$
R_{i j(i), 00}=\left[\begin{array}{cc}
\left(\bar{p}_{i}-\bar{p}_{j(i)}\right)^{T} & \left(\bar{p}_{j(i)}-\bar{p}_{i}\right)^{T} \\
0 & 0
\end{array}\right] .
$$

For a LRF formation, the leader equations are of course

$$
\left[\begin{array}{l}
\dot{\delta} x_{n} \\
\dot{\delta} y_{n}
\end{array}\right]=0
$$

Putting the equations together, we have for LRF

$$
\dot{\delta} p=K R_{e}^{-1}\left[\begin{array}{l}
R \\
0
\end{array}\right] \delta p
$$

and we have for coleaders

$$
\dot{\delta} p=K R_{e}^{-1}\left[\begin{array}{c}
R_{1, n-3} \\
r_{n-2} \\
0 \\
r_{n-1} \\
0 \\
r_{n} \\
0
\end{array}\right] \delta p
$$

where $K=\operatorname{diag}\left[K_{1}, \ldots, K_{n-1}, 0\right]$ with $2 \times 2 K_{i}$ to be specified, $R_{e}=\operatorname{diag}\left[R_{e 1}, \ldots, R_{e, n-1}, I_{2}\right]$ with each $R_{e i}$ being a $2 \times$ 2 submatrix of the rigidity matrix $R \in \Re^{2 n-3 \times 2 n}$. For coleaders $\left[R_{1, n-3}^{T}, r_{n-2}^{T}, r_{n-1}^{T}, r_{n}^{T}\right]^{T} \in \Re^{2 n-3 \times 2 n}$ is the rigidity matrix.

\section{A Reduced-Order System for LRF Formations}

For LRF formations, we define a reduced-order system by neglecting the stationary leader dynamics since $\dot{p}_{n}(t)=0$. Let the global coordinate basis have the leader at the origin and let the $x$-axis be an arbitrary direction. Let $z=\left[p_{1}, \ldots, p_{n-1}\right]^{T} \in$ $\Re^{2 n-2}, \bar{z}=\left[\bar{p}_{1}, \ldots, \bar{p}_{n-1}\right]$, and $z=\delta z+\bar{z}$ where $\delta z$ is assumed to be small. The reduced-order nonlinear system may then be written in the form

$$
\dot{z}=\bar{f}(z)
$$

The rigidity function associated with (24) is $r_{z}(z)=$ $\left[\ldots,\left\|z_{j}-z_{k}\right\|^{2}, \ldots\right]^{T}$ where the $i$ th entry of $r_{z}$ corresponds to an edge $e_{i} \in E$ connecting two vertices $j$ and $k$. If a vertex $l$ is connected to the leader, then the corresponding entry in $r_{z}$ is $\left\|z_{l}\right\|^{2}$. The equilibrium manifold associated with (24) is given by

$$
\Psi_{z}=\left\{z \in \Re^{2 n-2} \mid z=r_{z}^{-1}(d)\right\}
$$

$\Psi_{z}$ is a one-dimensional manifold that can be characterized by a rotation around the leader since the position of the leader is fixed. Therefore, since $\Psi_{z}$ is a closed and bounded subset of Euclidean space, it is compact.

Now, expanding in a Taylor series about the equilibrium position, we can rewrite (24) near the equilibrium position in the form

$$
\dot{\delta} z=J_{\bar{f}}(\bar{z}) \delta z+g(\delta z)
$$

where the first term represents the reduced-order linear system and the second term represents the nonlinear part of order two or higher. The reduced-order linear system may be written in the form

$$
\dot{\delta} z=\tilde{K} \tilde{R}_{e}^{-1}\left[\begin{array}{c}
\tilde{R} \\
0
\end{array}\right] \delta z
$$

where

$$
\tilde{K}=\operatorname{diag}\left[K_{1}, \ldots, K_{n-1}\right], \tilde{R}_{e}=\operatorname{diag}\left[R_{e 1}, \ldots, R_{e, n-1}\right]
$$


and $\tilde{R}$ is the submatrix of the rigidity matrix with the last two columns associated with the leader removed.

Observe that the Jacobian $J_{\bar{f}}(\bar{z})$ is rank deficient by one because of the row of zeros below the rigidity matrix. Thus, one of its eigenvalues is zero. Thus, the equilibrium position is nonhyperbolic, and we can apply center manifold theory as developed in Section II to try to determine local stability of the equilibrium position. Since $J_{\bar{f}}(\bar{z})$ has one zero eigenvalue, there exists an invertible matrix $Q$ such that

$$
Q J_{\bar{f}}(\bar{z}) Q^{-1}=\left[\begin{array}{cc}
0 & 0 \\
0 & A_{s}
\end{array}\right]
$$

where $A_{s} \in \Re^{2 n-3 \times 2 n-3}$ is a nonsingular matrix. Let $[\theta, \rho]^{T}=$ $Q \delta z$ where $\theta \in \Re$ and $\rho \in \Re^{2 n-3}$. Then (26) can be written in the form

$$
\begin{aligned}
& \dot{\theta}=g_{1}(\theta, \rho) \\
& \dot{\rho}=A_{s} \rho+g_{2}(\theta, \rho)
\end{aligned}
$$

where $g_{1}$ is the first entry of $Q g\left(Q^{-1}[\theta, \rho]^{T}\right)$ and satisfies $g_{1}(0,0)=0$ and $J_{g 1}(0,0)=0$, and $g_{2}$ is the last $2 n-3$ entries of $Q g\left(Q^{-1}[\theta, \rho]^{T}\right)$ and satisfies $g_{2}(0,0)=0$ and $J_{g 2}(0,0)=0$. This is in the normal form for center manifold theory.

To apply Theorem 5 we simply need the matrix $A_{s}$ to be Hurwitz. Here, due to cycles in the graph presenting the possibility of instability, $A_{s}$ must be made Hurwitz by a suitable choice of the gain matrices $K_{1}, \ldots, K_{n-1}$. It is not however obvious that this can be done, or how to do it.

\section{Full-Order System for Coleader Formations}

Expanding in a Taylor series about an equilibrium position, we can express (17) in the form

$$
\dot{\delta} p=J_{f}(\bar{p}) \delta p+g_{C}(\delta p)
$$

where the first term represents the linearized system given by (23) and the second term represents the nonlinear part of order two or higher. The Jacobian $J_{f}(\bar{p})$ is rank deficient by three because of the three rows of zeros; consequently, three of its eigenvalues are zero. Thus the equilibrium position is nonhyperbolic. Since $J_{f}(\bar{p})$ has three zero eigenvalues, there exists an invertible matrix $Q_{C}$ such that

$$
Q_{C} J_{f}(\bar{p}) Q_{C}^{-1}=\left[\begin{array}{cc}
0 & 0 \\
0 & A_{s C}
\end{array}\right] .
$$

where $A_{s C} \in \Re^{2 n-3 \times 2 n-3}$ is a nonsingular matrix. Let $\left[\theta_{C}, \rho_{C}\right]^{T}=Q_{C} \delta p$ where $\theta_{C} \in \Re^{3}$ and $\rho_{C} \in \Re^{2 n-3}$. Then (30) can be expressed in the form

$$
\begin{aligned}
& \dot{\theta_{C}}=g_{1 C}\left(\theta_{C}, \rho_{C}\right) \\
& \dot{\rho_{C}}=A_{s C} \rho+g_{2 C}\left(\theta_{C}, \rho_{C}\right)
\end{aligned}
$$

where $g_{1 C}$ comprises the first three entries of $Q_{C} g_{C}\left(Q_{C}^{-1}\left[\theta_{C}, \rho_{C}\right]^{T}\right)$ and satisfies $g_{1 C}(0,0)=0$ and $J_{g 1 C}(0,0)=0$, and $g_{2 C}$ comprises the last $2 n-3$ entries of $Q_{C} g_{C}\left(Q_{C}^{-1}\left[\theta_{C}, \rho_{C}\right]^{T}\right)$ and satisfies $g_{2 C}(0,0)=0$ and $J_{g 2 C}(0,0)=0$. This is in the normal form for center manifold theory.
Again, to apply Theorem 5 we simply need the matrix $A_{s C}$ to be Hurwitz, and due to cycles in the graph presenting the possibility of instability, $A_{s C}$ must be made Hurwitz by a suitable choice of the gain matrices $K_{1}, \ldots, K_{n}$. Showing that such a choice of gains is indeed possible for both LRF and coleader formations is the topic of the next section.

\section{Choosing Gains AND the Principal Minor Condition}

In this section, we show that it is possible to choose the gain matrices for each agent such that all nonzero eigenvalues of the linearized system have negative real parts. This is the case if a certain submatrix of the rigidity matrix has all leading principal minors nonzero. That this condition is satisfied by all LRF and coleader formations is shown in the following. The arguments are similar but not identical to those of [11].

\section{A. LRF Formations}

Let the gain matrices $K_{1}, \ldots, K_{n-1}$ be chosen as follows:

$$
K_{i}=\Lambda_{i} R_{e, i}, \quad i=1, \ldots, n-1
$$

where $\Lambda_{i}$ is a diagonal matrix. Then we have

$$
J_{\bar{f}}(\bar{z})=\Lambda\left[\begin{array}{c}
\tilde{R} \\
0
\end{array}\right]
$$

where $\Lambda \in \Re^{2 n-2 \times 2 n-2}$ is a diagonal matrix where the diagonal entries can be chosen independently. The linearized system then has the following form:

$$
\dot{\delta} z=\Lambda\left[\begin{array}{cc}
\hat{R} & r_{12} \\
0 & 0
\end{array}\right] \delta z=J_{\bar{f}}(\bar{z}) \delta z
$$

where $\Lambda \in \Re^{2 n-2 \times 2 n-2}$ is a diagonal matrix whose diagonal entries can be chosen independently and $\hat{R} \in \Re^{2 n-3 \times 2 n-3}$ is the rigidity matrix $R$ with the last three columns removed. We have the following result from [11].

Theorem 6 ([11]): Suppose $\hat{R}$ is a nonsingular matrix with every leading principal minor nonzero and let $\Lambda=\operatorname{diag}\left(\Lambda_{1}, \lambda_{2}\right)$ with $\Lambda_{1} \in \Re^{2 n-3 \times 2 n-3}$ diagonal and $\lambda_{2} \in \Re$. Then there exists a diagonal matrix $\Lambda_{1}$ such that the real parts of all nonzero eigenvalues of the linearized system are negative.

Thus, $2 n-3$ eigenvalues of $J_{\bar{f}}(z)$ have negative real part and clearly the remaining eigenvalue is zero due to the rank deficiency of $J_{\bar{f}}(z)$. To make use of Theorem 6 , we now need to show that $\hat{R}$ satisfies the principal minor condition for LRF formations. Let $V^{\prime}=\left\{v_{1}, \ldots, v_{n-2}\right\}$ represent the set of ordinary followers, and let agents $v_{n-1}$ and $v_{n}$ correspond to the remote follower and leader, respectively.

Theorem 7: Consider any minimally persistent LRF formation $F(G, p)$ of $n$ agents at generic positions in the plane. Then there exists an ordering of the vertices of $F$ and an ordering of the pair of outgoing edges for each vertex such that all leading principal minors of the associated $\hat{R}$ are generically nonzero.

The proof is nearly identical to the proof in [11]. We note the following partition of $\hat{R}$, which contrasts with the LFF partition in [11]: 


$$
\hat{R}=\left[\begin{array}{cc}
R\left(V^{\prime}\right) & \hat{r}_{12} \\
\hat{r}_{21}^{T} & \bar{x}_{n-1}-\bar{x}_{l}
\end{array}\right] .
$$

$R\left(V^{\prime}\right)$ is the principal submatrix of $\hat{R}$ obtained by retaining columns corresponding to the elements of $V^{\prime}$. The vector $\hat{r}_{21}^{T}$ has nonzero entries in the columns associated with $v_{l}$ and is zero elsewhere. The vector $\hat{r}_{12}$ has nonzero entries in the rows associated to edges that are pointing to the remote follower (there may be one or many nonzero entries), and is zero elsewhere. Additionally, consider a subset of ordinary follower vertices $V_{1} \subseteq V^{\prime}$ and define $R\left(V_{1}\right)$ as the principal submatrix of $\hat{R}$ obtained by retaining columns corresponding to the elements of $V_{1}$. We have the following result, which is stated in [11] for LFF formations and extends to LRF formations with identical proof.

Lemma 2: For any minimally persistent LRF formation, $\hat{R}$ is generically nonsingular and $R\left(V_{1}\right)$ is generically nonsingular for every $V_{1} \subseteq V^{\prime}$.

Remark: The proof of Lemma 2 for the LFF case in [11] is stated for $V_{1} \subset V^{\prime}$ and trivially extends to the case for $V_{1} \subseteq V^{\prime}$.

Lemma 2 establishes that $\hat{R}$ is generically nonsingular and that all even-order principal minors are nonzero. Finally, the proof that all odd order principal minors are also nonzero relies on an appropriate ordering of edges and is identical to the proof in [11].

This result shows that one can choose the diagonal matrix $\Lambda$ such that the real parts of all nonzero eigenvalues of the reduced-order linearized system (27) are negative (and accordingly the matrix $A_{s}$ in (29) is Hurwitz). The stabilizing gains are designed for a particular equilibrium point in the equilibrium manifold $\Psi_{z}$. It is important to note here that the control gains proposed in (33) may not be stabilizing for all other points in $\Psi_{z}$. Theorem 5 can be directly applied to show that for each $\bar{z} \in \Psi_{z}$, there is a neighborhood $\Omega(\bar{z})$ of $\bar{z}$ such that for any initial formation position $z(0) \in \Omega(\bar{z})$ there is a point $z^{*} \in \Psi$ such that $\lim _{t \rightarrow \infty} z(t)=z^{*}$ at an exponential rate, i.e., the formation converges locally exponentially to the desired shape.

\section{B. Coleader Formations}

Let the gain matrices $K_{1}, \ldots, K_{n}$ be chosen as follows:

$$
K_{i}=\Lambda_{i} R_{e, i}, \quad i=1, \ldots, n
$$

where the $\Lambda_{i}$ are diagonal matrices. Reorder the coleader coordinates as

$q=\left[x_{1}, y_{1}, \ldots, x_{n-3}, y_{n-3}, x_{n-2}, x_{n-1}, y_{n}, y_{n-2}, y_{n-1}, x_{n}\right]^{T}$.

An equilibrium position $\bar{q}$ is defined from $\bar{p}$ in the same manner as $q$ is defined from $p$. Then the linearized system has the form

$$
\dot{\delta} q=\Lambda\left[\begin{array}{cc}
\hat{R}_{C} & R_{12} \\
0 & 0
\end{array}\right] \delta q=J_{q}(\bar{q}) \delta q
$$

where $\Lambda \in \Re^{2 n \times 2 n}$ is a diagonal matrix whose diagonal entries can be chosen independently and $\hat{R}_{C} \in \Re^{2 n-3 \times 2 n-3}$ is a submatrix of the rigidity matrix $R$ that we will now define. Recall that $R$ has two columns associated with each agent: one comprised of $x$-coordinates and one of $y$-coordinates. The matrix $\hat{R}_{C}$ is obtained by removing the three columns from the rigidity matrix $R$ as follows: one associated with each coleader and not all of $x$ or $y$-type (i.e., one must remove two $x$-type and one $y$-type or vice versa). Theorem 6 extends to the coleader case; in particular, if $\hat{R}_{C}$ is nonsingular with every leading principal minor nonzero, then $\Lambda$ can be chosen such that all nonzero eigenvalues of the linearized system have negative real parts. Exactly three eigenvalues are necessarily zero. Again, to make use of this fact we now need to show that $\hat{R}_{C}$ satisfies the principal minor condition for all coleader formations. Let $V^{\prime}=$ $\left\{v_{1}, \ldots, v_{n-3}\right\}$ represent the set of ordinary followers, and let agents $v_{n-2}, v_{n-1}$, and $v_{n}$ correspond to the coleaders. We have the following result.

Theorem 8: Consider any minimally persistent coleader formation $F(G, p)$ of $n$ agents at generic positions in the plane. Then there exists an ordering of the vertices of $F$ and an ordering of the pair of outgoing edges for each vertex such that all leading principal minors of the associated $\hat{R}_{C}$ are generically nonzero.

We note the following structure of $\hat{R}_{C} \in \Re^{2 n-3 \times 2 n-3}$

$$
\hat{R}_{C}=\left[\begin{array}{cc}
R\left(V^{\prime}\right) & \hat{R}_{12} \\
\hat{R}_{21} & \hat{R}_{22}
\end{array}\right] .
$$

$R\left(V^{\prime}\right) \in \Re^{2 n-6 \times 2 n-6}$ is defined as before. Also, consider a subset of ordinary follower vertices $V_{1} \subseteq V^{\prime}$ and define $R\left(V_{1}\right)$ as before. Again, we have the following result from [11], which is stated for LFF formations and also extends to coleader formations with identical proof.

Lemma 3: For any minimally persistent coleader formation, $\hat{R}_{C}$ is generically nonsingular, and $R\left(V_{1}\right)$ is generically nonsingular for every $V_{1} \subseteq V^{\prime}$.

Lemma 3 establishes that the largest leading principal minor is generically nonzero and that all even order leading principal minors up to size $2 n-6$ are generically nonzero. The proof that all odd order leading principal minors up to size $2 n-7$ are also generically nonzero relies on an appropriate ordering of edges and is identical to the proof in [11]. It now remains to show that the second and third largest leading principal minors (of size $2 n-5$ an $2 n-4$ ) are generically nonzero. We have the following two results that treat separately the case where the coleaders are connected and the case where the coleaders are not connected.

Lemma 4: Suppose at most one coleader has its outgoing edge to $V^{\prime}$, the set of ordinary followers. Then the second and third largest leading principal minors of $\hat{R}_{C}$ are generically nonzero.

Proof: If at most one coleader, say that labeled $n$, has its outgoing edge to $V^{\prime}$, then another coleader, say that labeled $n-$ 1 , has its outgoing edge to $n$, and the remaining coleader, labeled $n-2$, has its outgoing edge to either $n-1$ or $n$ (suppose without loss of generality it is to $n-1$ ). This situation is illustrated in Fig. 4. Then the second and third largest leading principal submatrices of $\hat{R}_{C}$ have the structure

$$
\begin{aligned}
& M_{2 n-5}=\left[\begin{array}{cc}
R\left(V^{\prime}\right) & \times \\
0 & x_{n-2}-x_{n-1}
\end{array}\right] \\
& M_{2 n-4}=\left[\begin{array}{cc}
M_{2 n-5} & \times \\
0 & x_{n-1}-x_{n}
\end{array}\right]
\end{aligned}
$$

where $\mathrm{X}$ is a "don't care" vector (only $v_{n}$ may have an edge to $\left.V^{\prime}\right)$. Since $R\left(V^{\prime}\right)$ is generically nonsingular, then $M_{2 n-5}$ 


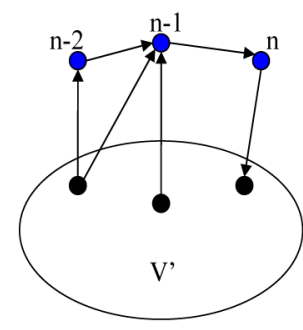

Fig. 4. Coleader formation with only one coleader having its outgoing edge to the set of ordinary followers $V^{\prime}$. There may be other edges from $V^{\prime}$ to the coleaders.

is generically nonsingular, which then implies that $M_{2 n-4}$ is generically nonsingular.

Before considering the case where the coleaders are not connected, we shall need the following result.

Lemma 5: Let $G(V, E)$ be the graph of a minimally persistent coleader formation with $n$ agents and denote the coleaders $n-2$, $n-1$, and $n$. Suppose we obtain a new graph $G_{2}\left(V_{2}, E_{2}\right)$ as follows. Introduce two new agents labeled $n+1$ and $n+2$. Suppose that $n+1$ has only one outgoing edge and that to $n+2$; $n+2$ has no outgoing edge; $n-2$ (which was a coleader) has an additional outgoing edge to $n+1, n-1$ an additional edge to $n+2$ and $n$ an additional edge to either $n+1$ or $n+2$. Then $G_{2}$ is minimally persistent with LFF structure, with $n+2$ the leader and $n+1$ the first follower.

Proof: First, we show that the underlying undirected graph $G_{2}$ is minimally rigid. Since $G$ is minimally persistent, the underlying undirected graph is minimally rigid and satisfies the conditions of Theorem 1 with $|E|=2|V|-3$. To obtain $G_{2}$, we have added to $G$ two new vertices and four new edges; thus, $\left|E_{2}\right|=2\left|V_{2}\right|-3$ and so $G_{2}$ satisfies the first condition of Theorem 1 for minimum edge count. Further, it is easy to check that no induced subgraph of $G_{2}$ involving vertices $n+1$ and $n+2$ violates the second condition of Theorem 1 . Thus, the underlying directed graph of $G_{2}$ is minimally rigid.

Next, since $G$ is minimally persistent with coleader structure, the coleaders each have exactly one outgoing edge, and all remaining vertices have exactly two outgoing edges. To obtain

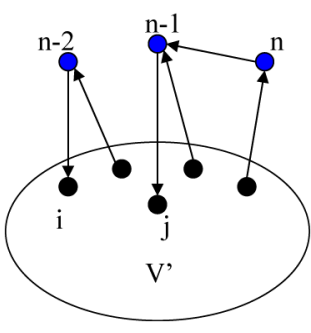

Fig. 5. Coleader formation with two coleaders having outgoing edges to the set of ordinary followers $V^{\prime}$. There may be other edges from $V^{\prime}$ to the coleaders.

$G_{2}$ we have added one outgoing edge from each coleader and so these vertices now have exactly two outgoing edges. Vertex $n+1$ has exactly one outgoing edge to $n$, and $n$ has no outgoing edges. Thus, $G_{2}$ is minimally persistent by Theorem 3 , and it has LFF structure with $n+2$ the leader and $n+1$ the first follower.

Now we have the following result where the coleaders are not connected.

Lemma 6: Suppose at least two coleaders have their outgoing edges to $V^{\prime}$, the set of ordinary followers. Then the second and third largest leading principal minors of $\hat{R}_{C}$ are generically nonzero.

Proof: Assume that coleaders labeled $n-2$ and $n-1$ have outgoing edges to vertices $i$ and $j$, respectively, both in $V^{\prime}$. This situation is illustrated in Fig. 5. Observe that the hypothesis permits $i=j$. The argument below applies regardless of whether $i=j$ and regardless of whether the sole outgoing edge of $n$ is to an element of $V^{\prime}$. Obtain a new LFF graph as described in Lemma 5 by introducing two artificial agents labeled $n+1$ and $n+2$. Call this new graph $G_{L F F}\left(V_{2}, E_{2}\right)$. Consider the matrices below where $B_{l}$ is the $l$ th-order principal submatrix of the matrix obtained by removing the three last columns of the rigidity matrix of the artificial graph of $G_{L F F}$, where the precise values of $a_{i}$ and $b_{i}$ are immaterial to the argument to be presented, as seen in the equation at the bottom of the next page. We note that all the even-dimensioned submatrices above are generically nonsingular, since they are the even-dimensioned leading principal submatrices of a LFF structure (see [11]).

$$
\begin{aligned}
& B_{2 n-5}=\left[\begin{array}{cc}
R\left(V^{\prime}\right) & a_{1} \\
b_{1} & x_{n-2}-x_{i}
\end{array}\right] \\
& B_{2 n-4}=\left[\begin{array}{ccc}
R\left(V^{\prime}\right) & a_{1} & a_{2} \\
b_{1} & x_{n-2}-x_{i} & y_{n-2}-y_{i} \\
0 & x_{n-2}-x_{n+1} & x_{n-2}-x_{n+1}
\end{array}\right] \\
& B_{2 n-3}=\left[\begin{array}{cccc}
R\left(V^{\prime}\right) & a_{1} & a_{2} & a_{3} \\
b_{1} & x_{n-2}-x_{i} & y_{n-2}-y_{i} & 0 \\
0 & x_{n-2}-x_{n+1} & x_{n-2}-x_{n+1} & 0 \\
b_{2} & 0 & 0 & x_{n-1}-x_{j}
\end{array}\right] \\
& B_{2 n-2}=\left[\begin{array}{ccccc}
R\left(V^{\prime}\right) & a_{1} & a_{2} & a_{3} & a_{4} \\
b_{1} & x_{n-2}-x_{i} & y_{n-2}-y_{i} & 0 & 0 \\
0 & x_{n-2}-x_{n+1} & x_{n-2}-x_{n+1} & 0 & 0 \\
b_{2} & 0 & 0 & x_{n-1}-x_{j} & y_{n-1}-y_{j} \\
0 & 0 & 0 & x_{n-1}-x_{n+2} & y_{n-1}-y_{n+2}
\end{array}\right]
\end{aligned}
$$


The third largest leading principal submatrix of $\hat{R}_{C}$ is given by $M_{2 n-5}=B_{2 n-5}$. Suppose $B_{2 n-5}$ is not generically nonsingular. Then it is singular everywhere. Then because of the underlying symmetry of the $x$ and $y$ columns, the same would be true of the matrix

$$
\left[\begin{array}{cc}
R\left(V^{\prime}\right) & a_{2} \\
b_{1} & y_{n-2}-y_{i}
\end{array}\right]
$$

But this implies that $B_{2 n-4}$ is singular, which establishes a contradiction. Therefore, $M_{2 n-5}$ is generically nonsingular.

The second largest leading principal submatrix of $\hat{R}_{C}$ is

$$
M_{2 n-4}=\left[\begin{array}{ccc}
R\left(V^{\prime}\right) & a_{1} & a_{2} \\
b_{1} & x_{n-2}-x_{i} & 0 \\
b_{2} & 0 & x_{n-1}-x_{j}
\end{array}\right] .
$$

We now argue that this matrix is generically nonsingular. Since $B_{2 n-2}$ is generically nonsingular, we assert the generic nonsingularity of $B_{2 n-3}$, which implies the generic nonsingularity of

$$
X=\left[\begin{array}{cccc}
R\left(V^{\prime}\right) & a_{1} & a_{2} & a_{3} \\
b_{1} & x_{n-2}-x_{i} & y_{n-2}-y_{i} & 0 \\
b_{2} & 0 & 0 & x_{n-1}-x_{j} \\
0 & x_{n-2}-x_{n+1} & x_{n-2}-x_{n+1} & 0
\end{array}\right]
$$

Indeed, suppose $B_{2 n-3}$ is singular for all positions. Then the symmetry of the $x$ and $y$ coordinates implies that the matrix obtained by replacing the last column of $B_{2 n-3}$, by the first four elements of the last column of $B_{2 n-2}$ must also be singular for all positions. Consequently $B_{2 n-2}$ must be singular, establishing a contradiction. Thus, $B_{2 n-3}$ is generically nonsingular and so is $X$.

Now we assert that $M_{2 n-4}$ is generically nonsingular. To establish a contradiction suppose $M_{2 n-4}$ is singular everywhere. Then again, the matrix obtained by replacing the second column of $M_{2 n-4}$ by the first three elements of the third column of $X$ must be singular everywhere. Thus, $X$ is singular. The contradiction proves the generic nonsingularity of $M_{2 n-4}$.

Thus, all leading principal minors of $\hat{R}_{C}$ are generically nonzero for all coleader formations. Therefore, again one can choose the diagonal matrix $\Lambda$ such that the real parts of all nonzero eigenvalues of the linearized system (38) are negative (and accordingly the matrix $A_{s C}$ in (32) is Hurwitz). The stabilizing gains are designed for a particular equilibrium point in the equilibrium manifold $\Psi$. It is important to note here that the control gains proposed in (37) may not be stabilizing for all other points in $\Psi$. Theorem 5 can be directly applied to show that for each $\bar{p} \in \Psi$, there is a neighborhood $\Omega(\bar{p})$ of $\bar{p}$ such that for any initial formation position $p(0) \in \Omega(\bar{p})$ there is a point $p^{*} \in \Psi$ such that $\lim _{t \rightarrow \infty} p(t)=p^{*}$ at an exponential rate, i.e., the formation converges locally exponentially to the desired shape.

Remark: There is an important distinction to be made between decentralized design and decentralized implementation. The control laws in this paper are based on minimally persistent information architectures, and selecting stabilizing gains requires a suitable ordering of the vertices and edges. Therefore, the design of our control laws is inherently centralized. However, persistent information architectures provide a basis from

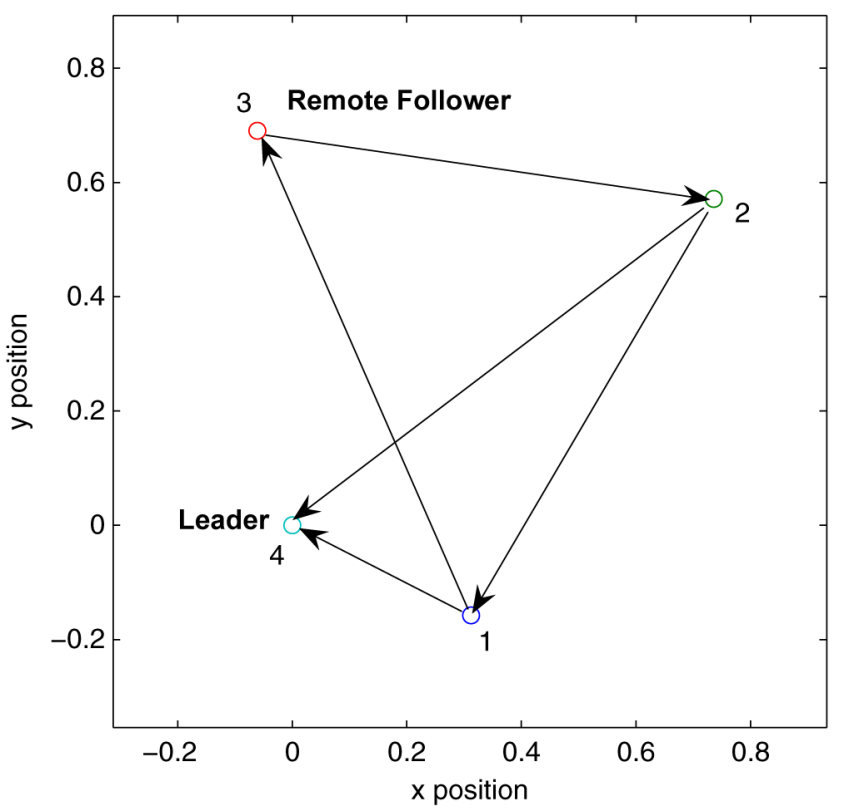

Fig. 6. LRF formation in unstable agent positions for identity gain.

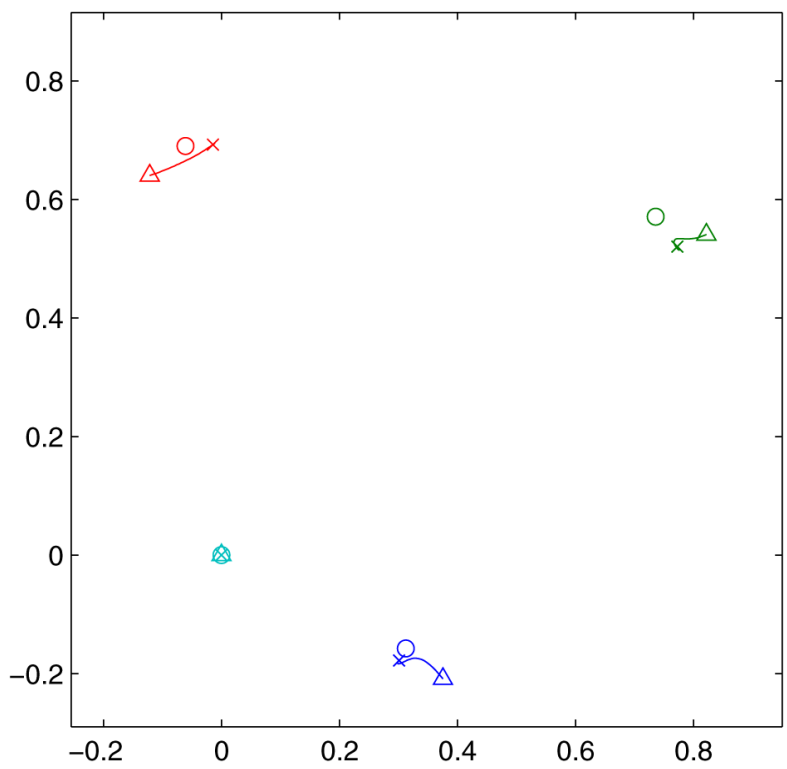

Fig. 7. Agent trajectories in the plane: the circles represent the initial desired formation shape, the triangles represent the perturbed agent positions, and the $X$ 's represent the final agents positions under the formation shape maintenance control laws. The desired shape has been restored. The leader does not move.

which we can design control laws with decentralized implementation. Once the design is established, our control laws require only local information.

\section{Simulation}

\section{A. LRF Formations}

In this section, we demonstrate the performance of our algorithm via simulation. Fig. 6 shows a LRF formation in the plane where agents 1 and 2 are ordinary followers, agent 3 is the remote follower, and agent 4 is the leader. Suppose the agents are in the desired formation shape in the position $\bar{p}=[0.3123,-0.1574,0.7359,0.5710,-0.0609,0.6901,0,0]$. 

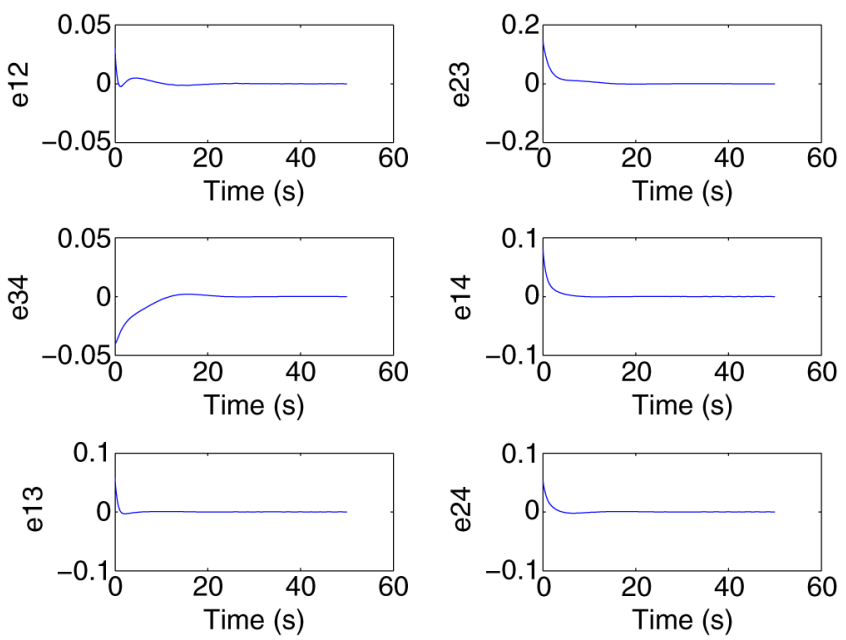

Fig. 8. Interagent distance errors, defined as $e_{i j}=\left\|p_{i}-p_{j}\right\|-d_{i j}$, all converge to zero, thus recovering the desired formation shape.

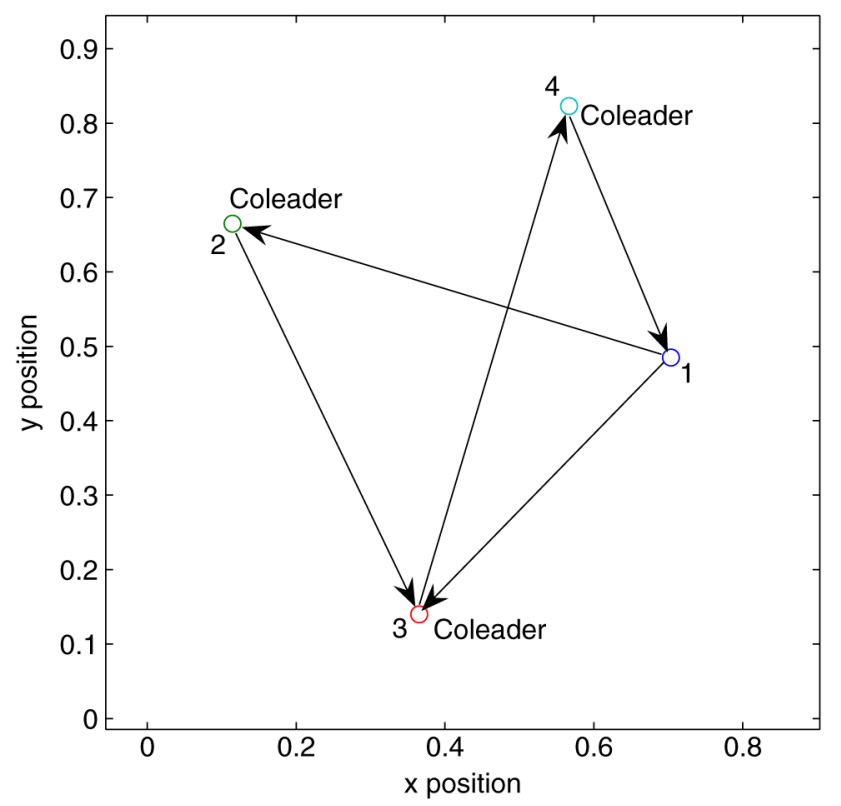

Fig. 9. Coleader formation in the plane in unstable agent positions for identity gain.

We note that if the gain matrices are all chosen to be identity, the nonzero eigenvalues of the linearized system are $\{-1.5363 \pm 0.9289 i, 0.0726,-1,-1\}$, which implies instability. Suppose the gain matrices are chosen with the structure given by (33) where the diagonal multipliers are $\Lambda_{1}=\operatorname{diag}[-2,2], \Lambda_{2}=\operatorname{diag}[-2,-1]$, and $\Lambda_{3}=\operatorname{diag}[2,0.5]$. Then the nonzero eigenvalues of the linearized system are given by $\{-1.9521 \pm 0.3196 i,-0.2521 \pm 0.3886 i,-0.7532\}$, and the desired formation shape is stable via the analysis in the previous sections. Fig. 7 shows the agent trajectories in the plane under the formation shape maintenance control laws. The desired formation shape is restored, though not to the initial unperturbed formation. Fig. 8 shows that the interagent distance errors all converge to zero. Simulations for large perturbations are explored for coleader formations.

\section{B. Coleader Formations}

Fig. 9 shows a coleader formation in the plane where agent 1 is an ordinary follower and agents

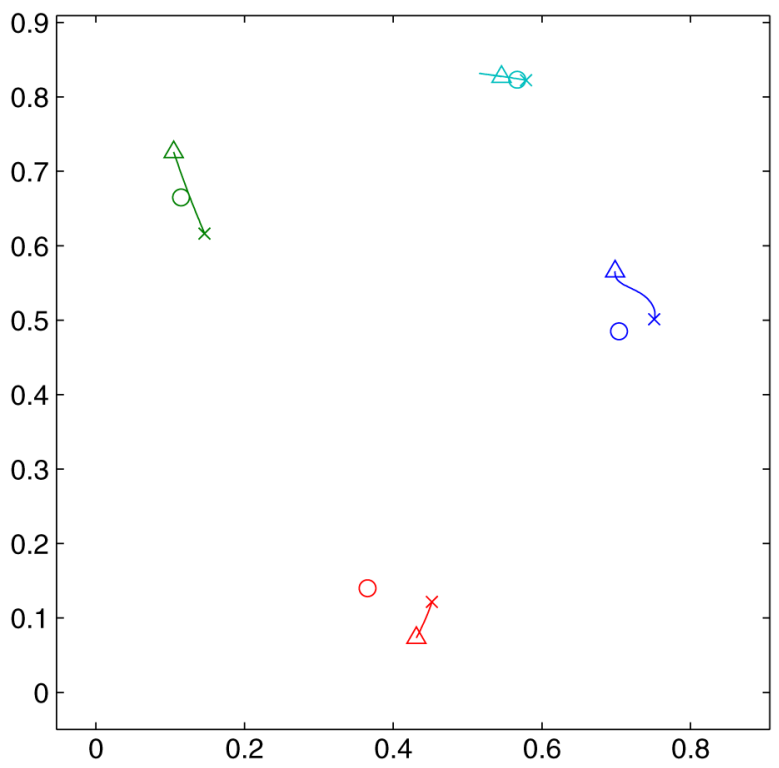

Fig. 10. Agent trajectories in the plane: the circles represent the initial desired formation shape, the triangles represent the perturbed agent positions, and the X's represent the final agents positions under the formation shape maintenance control laws. The desired shape has been restored, though the final formation is translated and rotated from the original unperturbed formation.
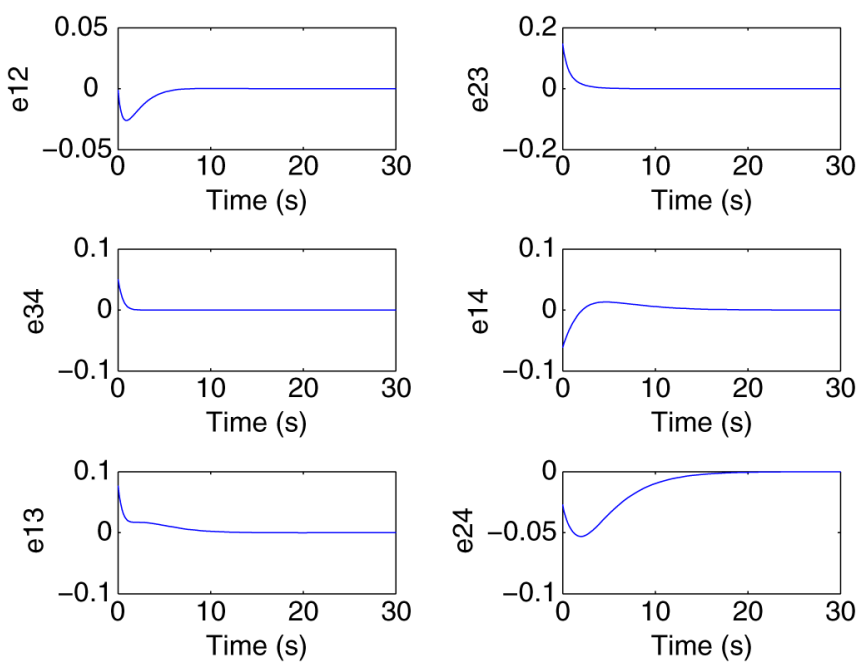

Fig. 11. Interagent distance errors all converge to zero, thus recovering the desired formation shape. That $e_{24}$, which does not correspond to an edge of the formation graph, goes to zero is due to the underlying rigidity and the convergence of the other $e_{i j}$ to zero.

2, 3, and 4 are coleaders. Suppose the agents are in the desired formation shape in the position $\bar{p}=$ $[0.7036,0.485,0.1146,0.6649,0.3654,0.140,0.5668,0.823]$. If the gain matrices are all chosen to be identity, the nonzero eigenvalues of the linearized system are $\{-1.2339 \pm 0.8376 i,-1.5356,-1,0.0034\}$, which implies instability. Suppose the gain matrices are chosen with the structure given by (37) where the diagonal multipliers are $\Lambda_{1}=\operatorname{diag}[-1.3,-0.9], \Lambda_{2}=\operatorname{diag}[0.5,0.75]$, $\Lambda_{3}=\operatorname{diag}[1.2,0.35]$, and $\Lambda_{4}=\operatorname{diag}[-2.7,-0.25]$. Then the nonzero eigenvalues of the linearized system are given by $\{-0.3655 \pm 0.0933 i,-0.5719,-0.9378,-1.0712\}$, and the desired formation shape is stable via the analysis in the previous sections. Fig. 10 shows the agent trajectories in the plane under the formation shape maintenance control laws. The 


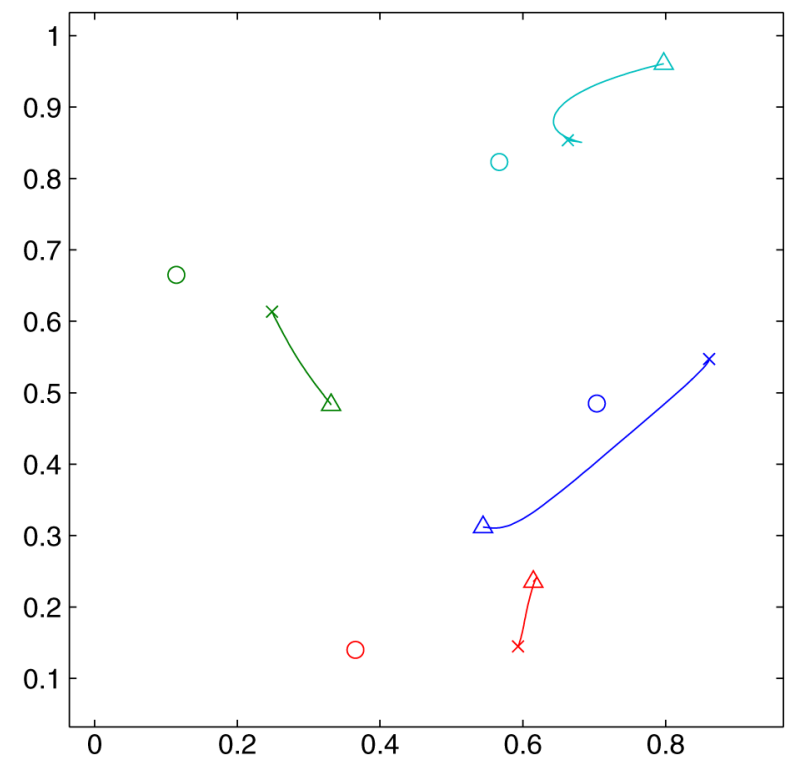

Fig. 12. Agent trajectories after a larger perturbations: the desired shape has been restored, though the final formation is translated and rotated from the original unperturbed formation. The circles, triangles and X's are as for Fig. 7.
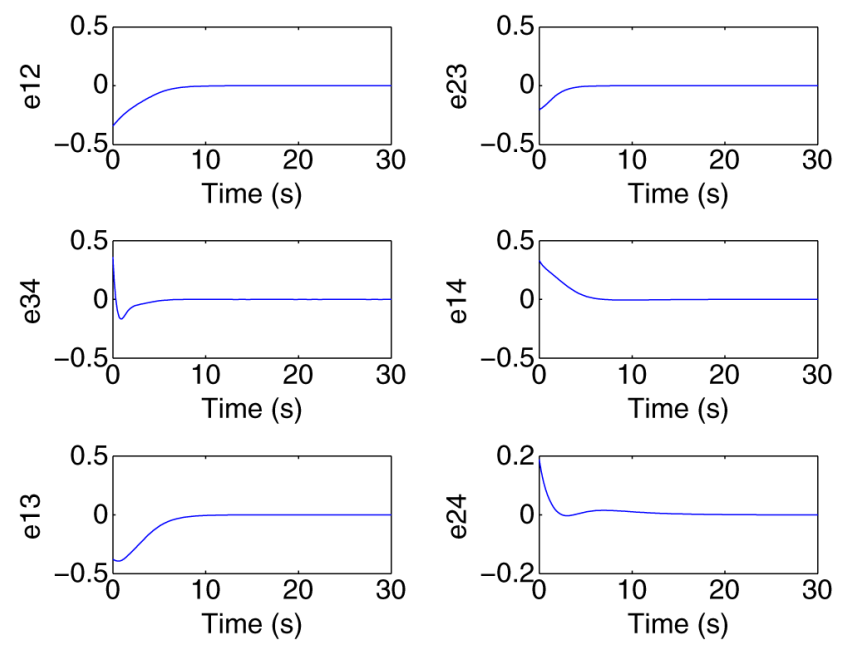

Fig. 13. Interagent distance errors all converge to zero, thus recovering the desired formation shape.

desired formation shape is restored, though the final formation is translated and rotated from the original unperturbed formation. Fig. 11 shows that the interagent distance errors all converge to zero.

The stability result is local; thus, convergence is only guaranteed for formations that are initially close enough to the desired shape. The following simulations investigate potential outcomes for larger perturbations from the desired shape. Fig. 12 shows convergence to the desired shape despite initial perturbations as large as $50 \%$ of the desired interagent distance, which indicates a sizable region of attraction. Fig. 13 shows the corresponding interagent distance errors converging to zero. Another possible outcome is shown in Fig. 14 where the agents converge to a formation that is equivalent, but not congruent, to the desired formation. In Fig. 15 all interagent distance errors corresponding to an edge in the graph converge to zero, whereas the only interagent distance error that does not correspond to an edge, viz. $e_{24}$, converges to a nonzero value. The position of agent 2 (green) has

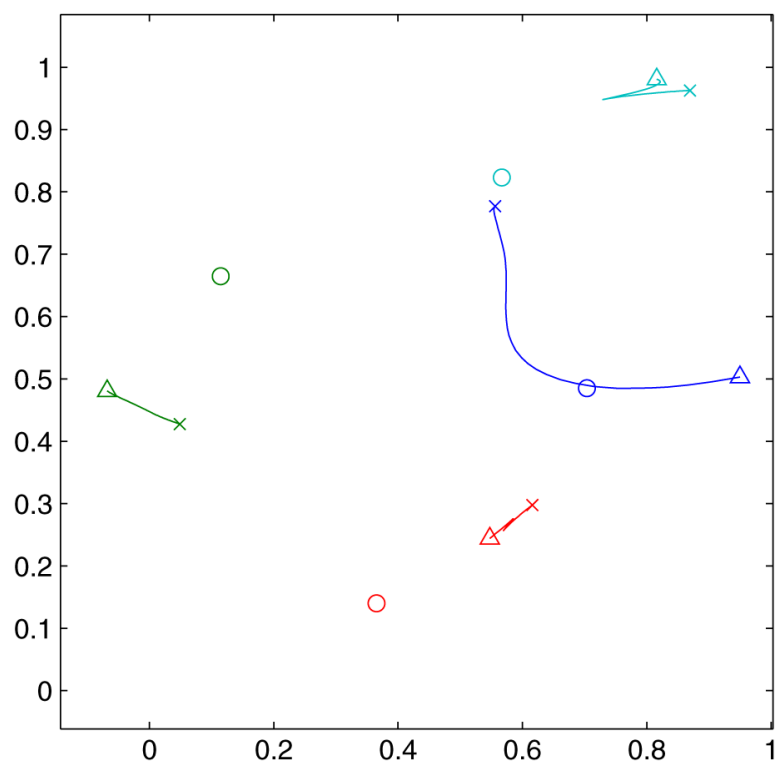

Fig. 14. Agent trajectories in the plane after large perturbations: the formation converges to an equivalent, but not congruent, shape.
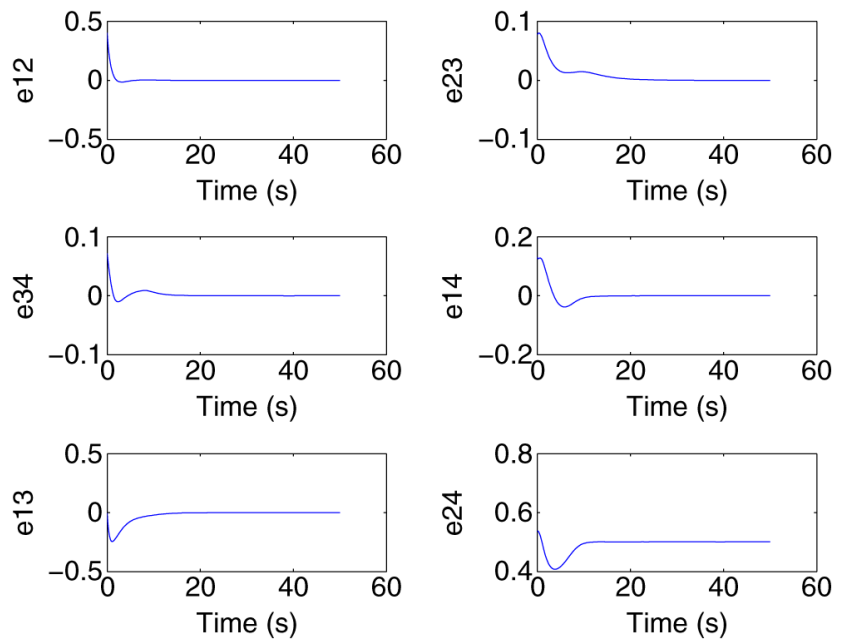

Fig. 15. All interagent distance errors except one converge to zero.

been reflected about the line connecting agents 1 and 3 (blue and red). Despite the fact that the linearized system is locally stable, we have observed instability in simulations for sufficiently large initial perturbations.

\section{CONCLUSION}

In this paper, we have addressed the $n$-agent formation shape maintenance problem for minimally persistent leader-remotefollower and coleader formations. We presented decentralized nonlinear control laws that restore the desired formation shape in the presence of small perturbations from the desired shape. The nonlinear system has a manifold of equilibria, which implies that the linearized system is nonhyperbolic. We applied center manifold theory to show local exponential stability of the equilibrium formation with desired shape. We have also shown that a principal minor condition holds for all LRF and coleader formations, which allows a choice of stabilizing gain matrices. Finally, we demonstrated our results through numerical simulation. 
There are many directions for future research. First, the stability results here are local, and an immediate task would be to determine the size of the region of attraction. Second, one can show that the stability properties of our control law are translationally, but not rotationally invariant. One could investigate whether or not it is possible to constrain the gain matrices in order to obtain rotational invariance. Preliminary calculations suggest that this will not always be possible. Third, non-minimally persistent formations will eventually be of interest because it may be desirable to control more than the minimum number of distances for formation shape maintenance in order to obtain a level of robustness. Finally, the impact of time-varying information architectures could be considered.

\section{REFERENCES}

[1] J. Fax and R. Murray, "Information flow and cooperative control of vehicle formations," IEEE Trans. Autom. Control, vol. 49, no. 9, pp. 1465-1476, Sep. 2004.

[2] W. Ren and R. Beard, "Consensus seeking in multiagent systems under dynamically changing interaction topologies," IEEE Trans. Autom. Control, no. 5, pp. 655-661, May 2005.

[3] W. Ren, R. Beard, and E. Atkins, "Information consensus in multivehicle cooperative control," IEEE Control Syst. Mag., vol. 27, no. 2, pp. 71-82, Apr. 2007.

[4] R. Olfati-Saber, J. Fax, and R. Murray, "Consensus and cooperation in networked multi-agent systems," Proc. IEEE, vol. 95, no. 1, pp. 215-233, Jan. 2007.

[5] T. Tay and W. Whiteley, "Generating isostatic frameworks," Structural Topol., vol. 11, pp. 21-69, 1985.

[6] T. Eren, P. Belhumeur, B. Anderson, and A. Morse, "A framework for maintaining formations based on rigidity," in Proc. 15th IFAC World Congr., Barcelona, Spain, 2002, pp. 2752-2757.

[7] R. Olfati-Saber and R. Murray, "Graph rigidity and distributed formation stabilization of multi-vehicle systems," in Proc. 41nd IEEE Conf. Decision Control, Las Vegas, NV, 2002, vol. 3, pp. 2965-2671.

[8] B. Anderson, C. Yu, S. Dasgupta, and A. Stephen Morse, "Control of a three-coleader formation in the plane," Syst. Control Lett., vol. 56, no. 9-10, pp. 573-578, 2007.

[9] M. Cao, C. Yu, A. Morse, B. Anderson, and S. Dasgupta, "Generalized controller for directed triangle formations," in Proc. IFAC World Congr., Seoul, Korea, 2008.

[10] M. Cao, B. Anderson, A. Morse, and C. Yu, "Control of acyclic formations of mobile autonomous agents," in Proc. 47th IEEE Conf. Decision Control, Cancun, Mexico, 2008, pp. 1187-1192.

[11] C. Yu, B. Anderson, S. Dasgupta, and B. Fidan, "Control of minimally persistent formations in the plane," SIAM J. Control Optim., vol. 48, no. 1, pp. 206-233, 2009.

[12] L. Krick, M. Broucke, and B. Francis, "Stabilization of infinitesimally rigid formations of multi-robot networks," Int. J. Control, vol. 82, no. 3, pp. 423-439, 2009.

[13] J. Baillieul and A. Suri, "Information patterns and Hedging Brockett's theorem in controlling vehicle formations," in Proc. 42nd IEEE Conf. Decision Control, Maui, HI, 2003, pp. 556-563.

[14] J. Hendrickx, B. Anderson, J. Delvenne, and V. Blondel, "Directed graphs for the analysis of rigidity and persistence in autonomous agent systems," Int. J. Robust Nonlinear Control, vol. 17, no. 10-11, pp. 960-981, 2007.
[15] C. Yu, J. Hendrickx, B. Fidan, B. Anderson, and V. Blondel, "Three and higher dimensional autonomous formations: Rigidity, persistence and structural persistence," Automatica, vol. 43, no. 3, pp. 387-402, 2007.

[16] L. Asimow and B. Roth, "The rigidity of graphs," Trans. Amer. Math Soc., pp. 279-289, 1978.

[17] L. Asimow and B. Roth, "The rigidity of graphs II," SIAM J. Appl. Math, vol. 68, pp. 171-190, 1979

[18] G. Laman, "On graphs and rigidity of plane skeletal structures," J. Eng Math., vol. 4, no. 4, pp. 331-340, 1970.

[19] J. Carr, Applications of Centre Manifold Theory. : : Springer, 1981.

[20] S. Sastry, Nonlinear Systems: Analysis, Stability, and Control. New York: Springer, 1999

[21] S. Wiggins, Introduction to Applied Nonlinear Dynamical Systems and Chaos. New York: Springer, 2003.

[22] I. Malkin, Theory of Stability of Motion. Washington, DC: United States Atomic Energy Commission, 1952.

[23] B. Aulbach, Continuous and Discrete Dynamics Near Manifolds of Equilibria. New York: Springer-Verlag, 1984.

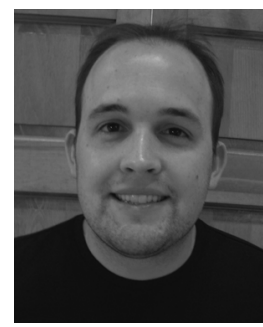

Tyler H. Summers (M'08) was born in Albuquerque, NM. He received the B.S. degree in mechanical engineering from Texas Christian University, Fort Worth, TX, in 2004 and the M.S. and $\mathrm{Ph} . \mathrm{D}$. degrees in aerospace engineering from the University of Texas at Austin in 2007 and 2010, respectively.

From September 2007 to December 2008, he was a Fulbright Scholar at the Australian National University, Canberra. He is currently with Automatic Control Laboratory, ETH Zürich, Zürich, Switzerland. He has held multiple research internships at Sandia National Laboratories and Air Force Research Laboratories. His research interests broadly include applications of feedback control theory to complex networks of dynamical systems, such as autonomous vehicle formations.

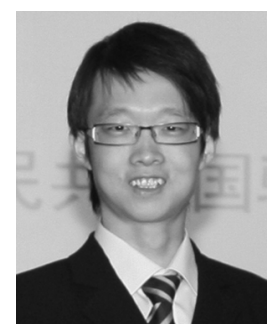

Changbin (Brad) Yu (M'08-SM'10) received the B.Eng. degree (first class honors) in computer engineering from Nanyang Technological University, Singapore in 2004 and the Ph.D. degree in information engineering from the Australian National University, Canberra, in 2008.

Since then he has been a Member of Academic Staff at the Australian National University and an Adjunct Researcher at National ICT Australia, Ltd. His current research interests include control of autonomous formations, multi-agent systems, mobile sensor networks, human-robot interaction and graph theory.

Dr. Yu had won a competitive Australian Postdoctoral Fellowship (APD) in 2007 and a prestigious Queen Elizabeth II Fellowship (QEII) in 2010. He was also a recipient of Australian Government Endeavour Asia Award (2005), Inaugural Westpac Australian Chinese Students New Age Award (2006), Chinese Government Outstanding Overseas Students Award (2006), Asian Journal of Control Best Paper Award (2006-2009), etc.

His current research interests include control of autonomous formations, multi-agent systems, mobile sensor networks, human-robot interaction and graph theory. 


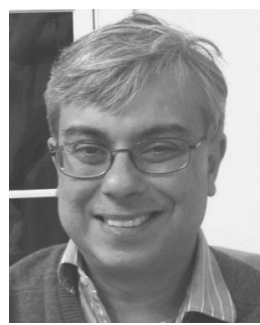

Soura Dasgupta (M'87-SM'93-F'98) was born in Calcutta, India, in 1959. He received the B.E. degree in electrical engineering from the University of Queensland, Brisbane, Australia, in 1980 and the $\mathrm{Ph} . \mathrm{D}$. in systems engineering from the Australian National University, Canberra, in 1985.

$\mathrm{He}$ is currently a Professor of electrical and computer engineering at the University of Iowa, Iowa City, IA. In 1981, he was a Junior Research Fellow in the Electronics and Communications Sciences Unit at the Indian Statistical Institute, Calcutta. He has held visiting appointments at the University of Notre Dame, University of Iowa, Université Catholique de Louvain-La-Neuve, Belgium, and the Australian National University. His research interests are in controls, signal processing, and communications.

Prof. Dasgupta served as an Associate Editor of the IEEE TRANSACTIONS ON AUTOMATIC CONTROL, IEEE Control Systems Society Conference Editorial Board, and the IEEE TRANS. CIRCUITS AND SYSTEMS-II from 1988 and 1991, 1998 to 2009, and 2004 and 2007 he, respectively. He is a corecipient of the Gullimen Cauer Award for the best paper published in the IEEE TRANSACTIONS ON CiRCUITS AND SYSTEMS in the calendar years of 1990 and 1991, a past Presidential Faculty Fellow, a subject editor for the International Journal of Adaptive Control and Signal Processing, and a member of the editorial board of the EURASIP Journal of Wireless Communications.

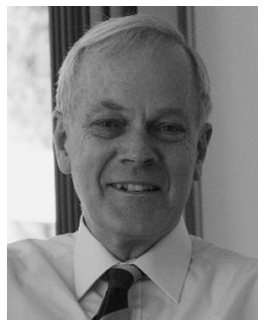

Brian D. O. Anderson (M'66-SM'74-F'75-LF'07) was born in Sydney, Australia. He studied mathematics and electrical engineering at Sydney University, Sydney, Australia, and received the $\mathrm{Ph} . \mathrm{D}$. degree in electrical engineering from Stanford University, Stanford, CA, in 1966.

$\mathrm{He}$ is a Distinguished Professor at the Australian National University, Canberra, and Distinguished Researcher in National ICT Australia. His current research interests are in decentralized control, sensor networks, and econometric modeling.

Prof. Anderson received the IEEE Control Systems Award of 1997, the 2001 IEEE James H. Mulligan, Jr., Education Medal, and the Bode Prize of the IEEE Control System Society in 1992. 